\title{
MODELLING MICROPOLAR BEHAVIOUR IN CORTICAL BONE
}

\author{
Jamie Frame (1), Dr. Marcus Wheel (2), Dr. Philip Riches (1)
}

\author{
1. Bioengineering Unit, University of Strathclyde, UK; 2. Department of Mechanical \\ Engineering, University of Strathclyde, UK
}

\section{Introduction}

Cortical bone is a heterogeneous material consisting of a hierarchical microstructure characterised by fibrous, porous and particulate features. This microstructure determines the macroscopic material properties. Bone prostheses are typically modelled using classical continuum elasticity however, the lack of microstructural characterisation may not adequately describe periprosthetic stress concentrations, with reduced ability in predicting failure. A material model which incorporates microstructural features may more accurately describe the material response (Fatemi, Van Keulen, \& Onck, 2002).

Micropolar elasticity includes a couple stress, in addition to the direct stresses, in its formulation. Micropolar materials exhibit a size effect in bending or torsion tests, where smaller specimens are relatively stiffer. Importantly, cortical bone has previously been shown to follow trends associated with micropolar behaviour (Yang, R. Lakes, 1982). We aim to include idealised Haversian canal structures into a FE model of bone to numerically assess potential micropolar behaviour.

\section{Methods}

Finite Element (FE) modelling (ANSYS 12.1) was used to create an array of regularly repeating voids in beams with and without surface perforations, crudely mimicking cortical bone's Haversian canal microstructure. By applying three-point-bending loading constraints (Figure 1) the stiffness of each beam can be compared over a range of depths, indicating the presence of a size effect in the material.

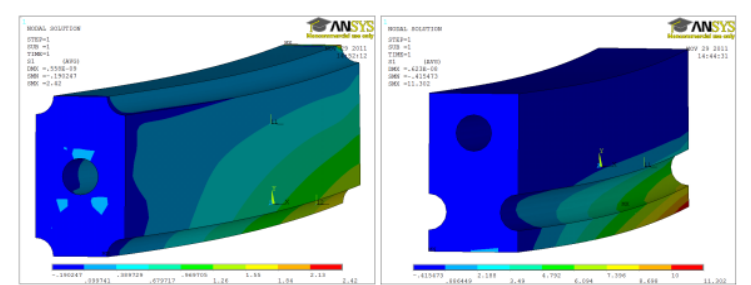

Figure 1: Three-point-bending loading for beams with and without surface perforations simplified to cantilever bending by applying symmetry

\section{Results and Discussion}

The idealised porous heterogeneous material demonstrated micropolar behaviour in 3-pointbending (Figure 2) with a size stiffening effect as the specimens become smaller. The micropolar characteristic length in bending was found to be dependent on the void arrangement and size.

In models with voids perforating the surface, an "anti-micropolar" size softening effect was observed, suggesting that the degree of surface roughness is of particular importance to the overall stiffness and in identifying micropolar behaviour. Moreover this indicates that surface effects are of significant importance in determining material properties in both FE and experimental methods.

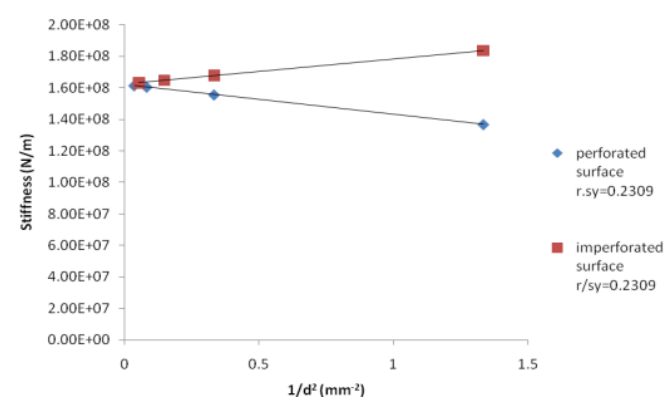

Figure 2: Plot of opposite size effects observed in beams with and without surface perforations

Initial experimental results from bovine cortical bone have been inconclusive, with both micropolar and anti-micropolar behaviour being observed. We hypothesise that surface roughness due to preparatory techniques may produce experimental artefacts are of the same order of magnitude as the micropolar effects thereby obscuring and confounding experimental results. Further work aims to control surface roughness in experimental tests to better characterise size effects and the micropolar behaviour of bone.

\section{References}

Fatemi, J., Van Keulen, F., \& Onck, P. Meccanica, 37(4), 385-396, 2002.

Yang, R. Lakes, J. Journal of Biomechanics, 15(2), 91-98, 1982 